P22 (continued)

characteristics were also assessed. Descriptive statistics were calculated. Monte Carlo Estimates for the Exact Test and ANOVA were used to assess statistical significance for the association between food security status and student characteristics. Statistical significance was considered $P<.05$.

Results: Among students in the sample, 51\% reported high food security, 24\% marginal food security, and 25\% food insecurity. Characteristics significantly associated with food security status were gender, year in school, having a car, and perceived health rating. Age, years in the US, academic performance, marital status, having dependent children, living on vs off-campus, employment status, receiving financial aid, weight status, cooking frequency, perceived cooking skills, and having a meal plan were not significantly associated with food security status.

Conclusions: Lack of food security is a serious issue among international students at the University of North Carolina at Chapel Hill, with nearly half reporting food insecurity or marginal food security. The characteristics identified in this study as being associated with food security status can help to inform future research and interventions for addressing food security status among international students.

Funding: University of North Carolina at Chapel Hill Food for All micro-grant.

\section{Food and Nutrition Policy}

\section{P23 Do Restaurants in Japan Support the Goals of Minimizing Food Loss and Maintaining Healthy Weight?}

Rie Akamatsu,DPH,RD, akamatsu.rie@ocha.ac.jp, Ochanomizu University, 2-1-1, Otsuka, Bunkyo-ku, Tokyo, Japan, 1128610

Background: Serving excessive amounts of food may not only stimulate overeating in customers but also increase leftovers. Minimizing food loss and having more people who maintain healthy weight are national goals in Japan and are included in the United Nations' Sustainable Development Goals (SDGs).

Objective: To examine how restaurants support the goals of minimizing food loss and maintaining healthy weight, and owners' thoughts about leftovers.

Study Design, Setting, Participants: A cross-sectional study was conducted via an internet survey through a Japanese research company. Four hundred and twelve restaurants owners participated.

Measurable Outcome/Analysis: Measures included frequency and record of leftovers, thoughts about serving amount and leftovers, readiness for achieving Japanese national goals, recognition and referring of SDGs, and demographics. Descriptive analysis was used.

Results: Three hundred seven out of 412 participants were men $(74.5 \%)$; average age was 51.0 years $(\mathrm{SD}=9.3)$. Most were small business owners $(n=357,86.7 \%)$; average number of seats was 32.3 ( $\mathrm{SD}=44.71)$. Twentyfive percent answered that leftovers were found "often" $(\mathrm{n}=5,1.2 \%)$ or "sometimes" $(\mathrm{n}=98,23.8 \%)$, but $68 \%$ $(n=281)$ answered that they did not record it regularly. Seventy-seven percent $(n=319)$ answered that the eating amount was affected by the serving amount but $61 \%$ $(n=253)$ thought that the customers should control their eating amount. Moreover, $84.2 \%$ believed that a lot of food is necessary for customer satisfaction $(n=347)$. Only few reported to have started something for achieving the national goals of minimizing food loss $(n=47,11.4 \%)$ and maintaining healthy weight $(\mathrm{n}=14,3.4 \%)$. The restaurants that referred to SDGs for running their business were only $2.7 \%(n=11)$, and $73.5 \%(n=303)$ answered never to have heard of SDGs.

Conclusions: Although a quarter of the restaurants had leftovers, most restaurant owners believed a large quantity food was needed. Moreover, very few restaurants knew about SDGs.

Funding: JSPS KAKENHI Grant Number JP18K02219.

\section{Behavior and Education Theory}

\section{P24 Differences in College Students' Decision Making Related to Sustainable Protein Choices}

Samantha Pender, Samantha_pender@my.uri.edu, University of Rhode Island, 69 Bristol Rd, Narragansett, RI, 02882; Beth Carlton, University of Rhode Island; Janette Bedoyan, $M S, B S, R D$, University of Rhode Island; Jade McNamara, PhD, RD, University of Maine; Geoffrey Greene, $P h D, L D N, R D$, University of Rhode Island

Background: Protein choices are an important component of the environmental cost of the food system. It is important to consider the limited research done on protein choice decision making in college students. Students who are aware of their protein choices at the decision point in college may be more likely to practice sustainable food choices throughout their life.

Objective: To compare demographic, attitudinal and behavioral variables in college students between those who consistently think about the environmental impact of their protein choices (C) and those who are less concerned (L).

Study Design, Setting, Participants: Participants, 1824 years old, consenting to participate in this cross-sectional survey $(n=390)$, were recruited from introductory classes at a Northeastern university for extra credit. Participants were categorized by their response to "How often do you think about the impact of meat consumption on the environment?" with responses of "always" or "vegetarian" categorized as C and "sometimes" or "never" categorized as L.

Measurable Outcome/Analysis: Group (C and L) was compared using chi-square or t-test analysis for demographics, stage of change for environmentally conscious protein choice (EC), and green eating (GE), red meat consumption, and health consciousness. 